\title{
Photochemical internalization and gemcitabine combined with first-line chemotherapy in perihilar cholangiocarcinoma: observations in three patients $\square$
}

\section{(ㄷ)(구)(우 $\odot$}

\author{
Authors \\ Institutions \\ 1 Dept. of Gastroenterology and Endocrinology, Klinikum \\ Nürnberg, Paracelsus Medical University, Nürnberg, \\ Germany \\ 2 Dept of Medical Oncology West German Cancer Center, \\ University Hospital Essen, Germany \\ $3 \mathrm{PCl}$ Biotech AS, Oslo, Norway \\ 4 Medical Department II, University Hospital, Ludwig \\ Maximilians-University, Munich, Germany \\ 5 Goethe University Medical Center, Frankfurt, Germany
}

Alexander Dechêne ${ }^{1}$, Stefan Kasper ${ }^{2}$, Hans Olivecrona ${ }^{3}$, Joerg Schirra ${ }^{4}$, Joerg Trojan ${ }^{5}$

submitted 6.3.2020

accepted after revision 17.8 .2020

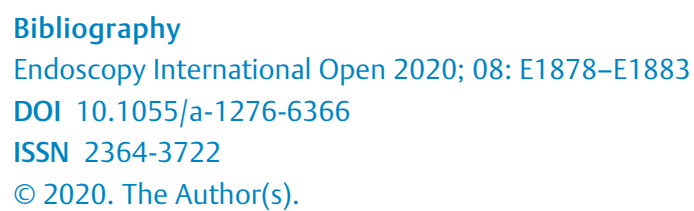

\section{ABSTRACT}

Photochemical internalization $(\mathrm{PCl})$ is a technology to induce a localized, intracellular enhancement of therapeutics that are processed through endosomal pathways, including gemcitabine in malignant cells. In addition to a direct phototoxic and tumoricidal effect, $\mathrm{PCl}$ specifically disrupts endosomal membranes and, thereby, the compartmentalization of certain cytotoxic compounds to enhance a drug's intended intracellular target reach within the tissue treated. Non-resectable extrahepatic cholangiocarcinoma (eCCA) is a common primary tumor and gemcitabine/cisplatin chemotherapy is widely considered standard of care for it. PCI is well suited as an endoscopic intervention, and clinical observations in three subjects participating in a phase I/Ila dose escalation safety trial are described. The trial included patients with perihilar, non-resectable CCA suitable for standard-of-care chemotherapy. Per protocol, a single endoscopic $\mathrm{PCl}$ procedure with gemcitabine was conducted at the initiation of standard gemcitabine/cisplatin therapy. Sixteen patients enrolled in the initial dose escalation phase of the trial, which later was extended to explore the safety of a second $\mathrm{PCl}$ procedure during chemotherapy.

While limited to a case series, the various clinical observations described here serve to illustrate the effects of localized, perihilar tumor targeting in appropriate patients by any safe methodology, including $\mathrm{PCl}$. As previously indicated by clinical data using other localized treatment modalities, adding a directed, tumor-targeting treatment to systemic therapy to ameliorate the progressively expanding extrahepatic tumor burden can have important effects on the overall outcome of systemic treatment in many patients who have incurable eCCA.

\section{Introduction}

Perihilar cholangiocarcinoma (CCA) is an adenocarcinoma with variable histological characteristics arising from the proximal portion of the common bile duct, including the main left or right hepatic ducts or their confluence. Surgical resection is the only curative option, but because early symptoms are infre- quent, most patients present with obstructive jaundice or cholangitis on the basis of a primarily non-resectable, advancedstage tumor and an expected survival of 6 to 12 months [1,2]. Standard of care is biliary drainage and systemic therapy, most often gemcitabine/cisplatin chemotherapy, resulting in a median overall survival of almost 12 months [3]. 
Based on recent developments in endoscopic interventions, various local tumor-targeting treatment modalities are used in specialized centers today. The common goal is to achieve tumor control and patency of the main bile ducts, as the consequences of biliary obstruction are a leading cause of both morbidity and mortality in this malignancy. Techniques include local ablation and embolization, brachytherapy, radiofrequency ablation and, significantly, photodynamic therapy (PDT).

PDT has a favorable safety profile and is a viable option for unresectable tumors and has been used as targeted, locoregional therapy for over two decades in CCA. PDT induces reactive oxygen species generation, leading to cell death and microvasculature damage, and it induces an inflammatory reaction locally that can translate to traceable systemic immune effects [4]. In several smaller trials, PDT has been shown to prolong survival in patients with non-resectable CCA and improve quality of life.

Many therapeutic compounds are entrapped in endosomes and further processed there without reaching their intracellular targets within the cytosol or the nucleus. Photochemical Internalization $(\mathrm{PCl})$ is a photochemical technology in clinical development in which a photochemical reaction is used to enhance the effect of such drugs by increasing their intracellular availability and facilitating their ability to reach the target [5]. A specific photosensitizer (TPCS 2 a, fimaporfin) administered systemically initially localizes to the outside of the cell membrane and thereafter translocates to reside inside the endosomal membrane by endocytosis ( $\triangleright$ Fig.1a). Laser illumination $(652 \mathrm{~nm})$ then induces a photochemical disruption of endocytic vesicles, dispersing its contents within cells, and also has PDTlike effects (> Fig. 1b).

The clinical efficacy of $\mathrm{PCl}$ was initially demonstrated in a phase I trial of $\mathrm{PCl}$ with bleomycin in patients with head and neck cancer [6]. Studies in vivo and in vitro (PCI Biotech, manuscript in preparation) showed that $\mathrm{PCl}$ strongly enhances the effect of the gemcitabine in cancer cells. The specific cellular uptake of gemcitabine are deranged in malignant cells, in which the drug is processed via endosomal pathways. Like PDT, PCI can be applied to extrahepatic CCA (eCCA) by use of an optic fiber for endoscopic illumination and localized therapy after systemic administration of the photosensitizer fimaporfin.

A phase I dose escalation trial (yet to be published) in 16 patients with non-resectable perihilar CCA was conducted using $\mathrm{PCl}$ with gemcitabine on a single occasion at the initiation of standard systemic therapy with gemcitabine/cisplatin for up to eight cycles, as in standard of care. The trial was extended $(\mathrm{N}=$ 7) to evaluate the safety of adding a second $\mathrm{PCl}$ procedure after four of the eight chemotherapy cycles. Here, the author's present three cases from this dose escalation trial (yet to be published) in patients with unresectable, perihilar CCA to illustrate clinical observations made in response to the treatment.
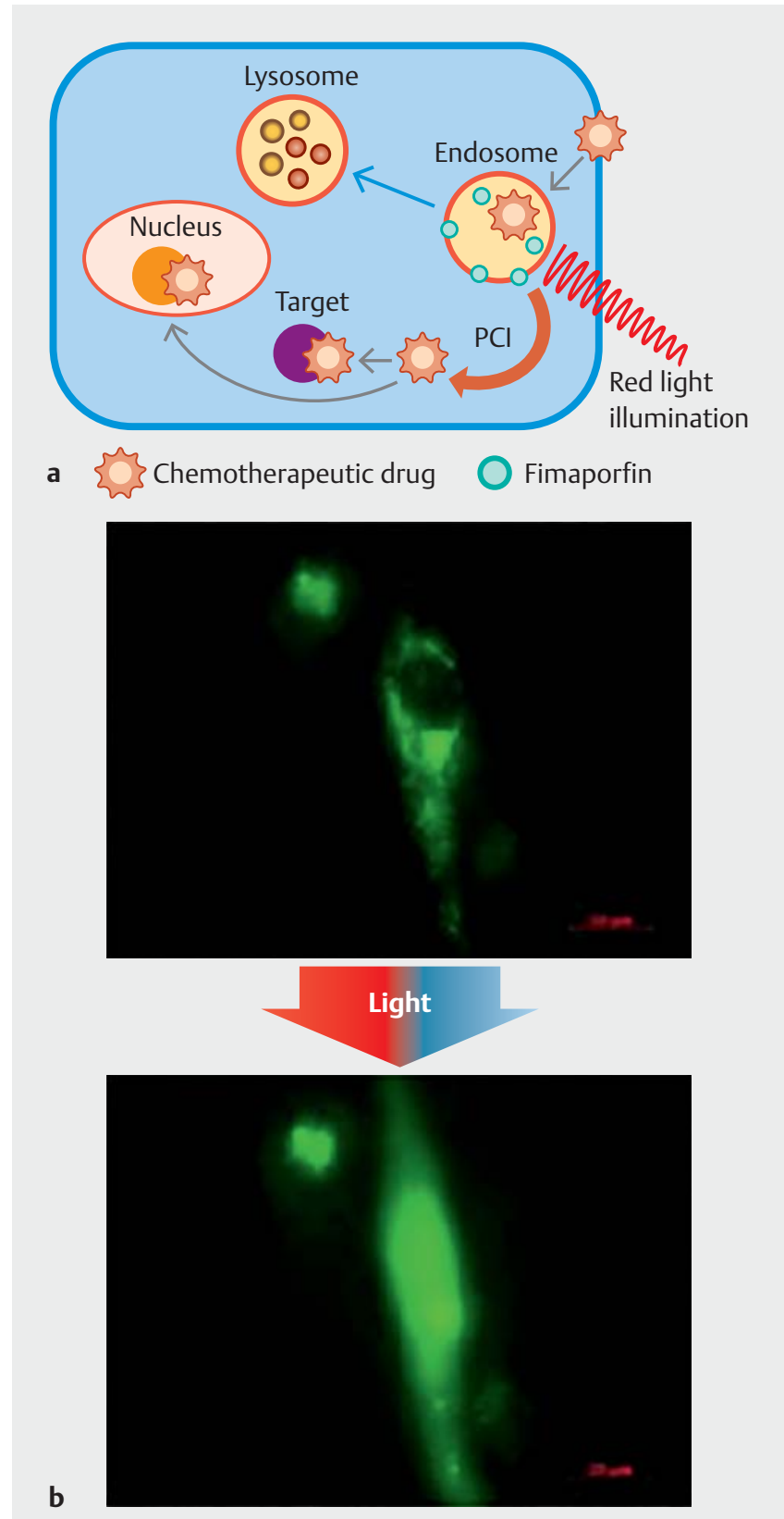

- Fig. 1 Photochemical internalization (PCI) mode of action. a After administration of the photosensitizer fimaporfin, due to its amphiphilic properties, it is incorporated in the cell membrane. After endocytosis, the molecule stays localized within the endosome membrane. Upon illumination (652 nM), reactive oxygen species are instantly formed, disrupting the endosome membrane, which allows for the escape into the cytosol of a variety of compounds, including a number of chemotherapeutic drugs. b PCI reaction effect under microscopy, showing disbursement of the Alexa488marked ovalbumin in vitro (Source: PCI Biotech; Photo: Dr Pål Kristian Selbo). 


\section{Patients and methods}

\section{$\mathrm{PCl}$ procedure}

The patients underwent endoscopic retrograde cholangiopancreatography (ERCP) 4 days after intravenous administration of fimaporfin, and 4 hours after gemcitabine infusion at standard dose on the day of $\mathrm{PCl}$ treatment. After removal of previously placed stents within the tumor area and under fluoroscopic guidance, a fiber-optic catheter with two radio-opaque markers delineating the cylindrical diffusor (light emitting) at its tip was advanced through the tumor surrounding the bile duct and retracted to an optimal position for illumination. A laser connected to the catheter was then activated to deliver the monochromatic (652 nm) light for approximately 5 minutes.

\section{Case reports}

Case 1

In October 2014, a 61-year-old male patient was diagnosed with unresectable perihilar CCA without metastatic or lymph node involvement. He was eligible for first-line standard gemcitabine/cisplatin chemotherapy and enrolled in the $\mathrm{PCl}$ dose escalation trial. In December 2014, he received PCI with fimaporfin at the lowest dose investigated in the trial $(0.06 \mathrm{mg} / \mathrm{kg})$ followed by gemcitabine and illumination at $30 \mathrm{~J} / \mathrm{cm}$, which he tolerated well. Subsequently, he completed eight cycles of gemcitabine/cisplatin as per study protocol with two treatment cycle delays due to cholangitis and neutropenia episodes. After 3 months, the patient's tumor biomarkers levels were decreased ( Table 1) and computed tomography (CT) scans demonstrated stable disease (SD) per the response evaluation criteria in solid tumors (RECIST) criteria until study end at Month 6.

In August 2016, a routine stent-exchange ERCP detected an elongation of the man's hilar stricture. CT scan confirmed progression with extraductal tumor expansion. At the patient's request, with all study measurements finalized and the study sponsor's consent, a second $\mathrm{PCl}$ treatment was conducted in September 2016, 20.5 months after the first treatment. The highest study dose, by then the recommended phase II dose $(0.25 \mathrm{mg} / \mathrm{kg}$ fimaporfin, light dose $30 \mathrm{~J} / \mathrm{cm})$, was used. No adverse events occurred, except an unplanned stent exchange for cholangitis in October 2016. The patient's disease was stable for another 22 months until July 2018, when tumor progression at the hepatic hilum and peritoneal spreading were detected. The patient received five cycles of gemcitabine/oxaliplatin before October 2018 and died the next month of cholangiosepticemia, 49 months after the diagnosis of primarily unresectable CCA (47 months after study enrollment). From the start of his treatment, a total of four unplanned stent exchanges were necessary due to non-severe cholangitis; still, the patient's overall quality of life was good.

Case 2

In January 2016, a 47-year-old male patient was diagnosed with perihilar cholangiocarcinoma (Bismuth Type IV) without radiologically metastatic disease or lymph node involvement. An explorative laparotomy showed peritoneal carcinomatosis, but as the man was otherwise healthy with functioning stents in both hepatic main ducts, he was enrolled in the PCI trial. Treatment was performed in March 2016 with fimaporfin at $0.25 \mathrm{mg} / \mathrm{kg}$, followed by gemcitabine and illumination at $30 \mathrm{~J} / \mathrm{cm}$. After the procedure, the patient had right-sided abdominal pain necessitating treatment with opioids, which resolved completely within 2 days. Six weeks after $\mathrm{PCI}$ treatment, he was readmitted and hospitalized with an episode of cholangitis. His left-sided stent was exchanged, while the right-sided internal stent had to be replaced with percutaneous cholangiodrainage. The patient's cholangitis quickly resolved and 4 weeks later, the percutaneous cholangiodrainage was removed and replaced with two internal stents again. The patient received all eight cycles of gemcitabine/cisplatin as per protocol.

At 3 months, an MRI demonstrated SD of the perihilar target lesion, and he was in partial remission in November 2016 at the end of the study, with decreased cancer antigen (CA) 19-9 levels ( $\triangleright$ Table 1 ). After the active study period, ERCPs were performed every 8 to 12 weeks for stent exchange. One episode of cholangitis occurred in November 2017. In December 2018 a metal stent was inserted. In January 2019, the patient's CA19-9 levels again were highly elevated and on MRI, disease progression with metastatic hepatic and peritoneal lesions was detected. Reintroduction of gemcitabine/cisplatin in April 2019 stabilized his disease, as reflected in MRI and CA19-9 levels. Chemotherapy was continued until July 2019, at which point the patient's Eastern Cooperative Oncology Group (ECOG) performance status was still 0 and his SD was confirmed radiologically. The patient was alive as of April 2020, but deteriorating with progressive disease.

Case 3

A 68-year-old male (ECOG 0-1) was diagnosed in November 2017 with a perihilar cholangiocarcinoma without detectable disease spread. The tumor was non-resectable at laparotomy, and the patient was enrolled in the PCI trial's extension. On MRI, a $30-\mathrm{mm}$ stenosis of the subhilar common bile duct (CBD) was visible on MRI that extended $8 \mathrm{~mm}$ apically into

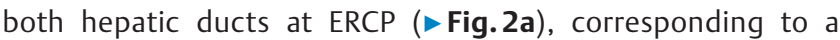
$22.5 \mathrm{~mm} \times 20.2 \mathrm{~mm}$ tumor surrounding the CBD (Bismuth IIla). Following $\mathrm{PCl}$ with gemcitabine in February 2018, the patient completed only three gemcitabine/cisplatin cycles due to progressive renal impairment and episodes of cholangitis.

Partial response of the target biliary tumor was noted at 3 months. At 5 months, despite abrogated chemotherapy, the patient consented to a second $\mathrm{PCI}$ treatment with gemcitabine per protocol. Four weeks later, the patient was hospitalized with cholangitis. On ERCP, an irregular necrotic stenosis consisting of a $4 \times 1 \mathrm{~cm}$ solid cast of necrosis in the CBD extending into both hepatic ducts ( $>$ Fig. $\mathbf{2 b}$ ) was removed by balloon extraction. The tumor location were thereafter visibly free from stenosis ( $>$ Fig. $2 c$ ).

After a confirmatory cholangioscopy 1 week later ( $\triangleright$ Fig. 2 d and Patient 3, - Video 1) the patient's ducts were left without drainage, with no signs of obstruction. Prescheduled ERCPs revealed a moderate stenosis recurrence, which led to reintroduction of biliary endoprostheses 5 weeks after the balloon ex- 
- Table 1 Patient characteristics during the active study period (6 months) and follow-up data.

\begin{tabular}{|c|c|c|c|c|c|c|}
\hline & \multicolumn{2}{|l|}{ Case 1} & \multicolumn{2}{|l|}{ Case 2} & \multicolumn{2}{|l|}{ Case 3} \\
\hline Gender & \multicolumn{2}{|l|}{ M } & \multicolumn{2}{|l|}{ M } & \multicolumn{2}{|l|}{ M } \\
\hline Age & \multicolumn{2}{|l|}{61} & \multicolumn{2}{|l|}{47} & \multicolumn{2}{|l|}{68} \\
\hline Lesion location & \multicolumn{2}{|c|}{ Perihilar: Bismuth IIIB } & \multicolumn{2}{|c|}{ Perihilar: Bismuth IV } & \multicolumn{2}{|c|}{ Perihilar: Bismuth IIIA } \\
\hline TNM stage & \multicolumn{2}{|l|}{ T4N0M0 } & \multicolumn{2}{|l|}{ TxNxM1 } & \multicolumn{2}{|l|}{ T2BNOM0 } \\
\hline Tumor marker ${ }^{1}$ & CA $19-9$ & CEA & CA 19-9 & CEA & CA $19-9$ & CEA \\
\hline - Baseline & 67.8 & 1.2 & 1898 & 2.9 & 83.1 & 3.0 \\
\hline - 3 months & 19.9 & 1.1 & 608 & 3.7 & 98.5 & 3.6 \\
\hline - Study end: 6 months & 22.5 & 0.8 & 136 & 3.5 & 105.0 & 4.6 \\
\hline \multirow[t]{7}{*}{ - Follow-up (month: result) } & 9 m: 18.5 & $9 \mathrm{m:} 1.6$ & 134 & 4.0 & \multirow[t]{7}{*}{ NR } & \multirow[t]{7}{*}{ NR } \\
\hline & $21 \mathrm{m:} 112.7^{2}$ & $21 \mathrm{m:} 1.4$ & NR & NR & & \\
\hline & $25 \mathrm{m:} 105.8$ & $25 \mathrm{m:} 1.9$ & NR & NR & & \\
\hline & $30 \mathrm{~m}: 67.2$ & $30 \mathrm{m:} 1.2$ & $25 \mathrm{~m}: 4766$ & $25 \mathrm{~m}: 8.4$ & & \\
\hline & 39 m: 315 & $39 \mathrm{m:} 2.5$ & 37 m: 3329 & 37 m: 9.4 & & \\
\hline & 44 m: 662.9 & $44 \mathrm{~m}: 2.4$ & $49 \mathrm{m:}>10000$ & $49 \mathrm{~m}: 30.0$ & & \\
\hline & $46 \mathrm{m:} 160.3$ & NR & & & & \\
\hline \multicolumn{7}{|l|}{ Chemo cycles on study: } \\
\hline - Gemcitabine & \multicolumn{2}{|c|}{$1000 \mathrm{mg} / \mathrm{m}^{2}: 6750 \mathrm{mg} / \mathrm{m}^{2}: 2$} & \multicolumn{2}{|l|}{$1000 \mathrm{mg} / \mathrm{m}^{2}: 7$} & \multicolumn{2}{|c|}{$1000 \mathrm{mg} / \mathrm{m}^{2}: 3$} \\
\hline \multirow{2}{*}{ - Cisplatin } & \multirow{2}{*}{\multicolumn{2}{|c|}{$25 \mathrm{mg} / \mathrm{m}^{2}: 8$}} & \multicolumn{2}{|l|}{$750 \mathrm{mg} / \mathrm{m}^{2}: 1$} & \multirow{2}{*}{\multicolumn{2}{|c|}{$25 \mathrm{mg} / \mathrm{m}^{2}: 3$}} \\
\hline & & & \multicolumn{2}{|l|}{$25 \mathrm{mg} / \mathrm{m}^{2}: 8$} & & \\
\hline \multirow[t]{2}{*}{$\begin{array}{l}\text { - Therapies after study } \\
\text { inclusion (months) }\end{array}$} & \multicolumn{2}{|c|}{$\begin{array}{l}\text { Month 21: PCl with gemcitabine } \\
1000 \mathrm{mg} / \mathrm{m}^{2}\end{array}$} & $\begin{array}{l}\text { Months } 35-42 \\
\text { platin }\end{array}$ & mcitabine/cis- & No & \\
\hline & $\begin{array}{l}\text { Month } 30-33 \\
\text { oxaliplatin (st }\end{array}$ & $\begin{array}{l}\text { citabine/ } \\
\text { d dose) }\end{array}$ & & & & \\
\hline PCl treatment(s) & 1 st & 2nd (off study) ${ }^{2}$ & $1 \mathrm{st}$ & 2nd & $1 \mathrm{st}$ & $\begin{array}{l}\text { 2nd (on study in } \\
\text { Phase lla }\end{array}$ \\
\hline - Light dose & $30 \mathrm{~J} / \mathrm{cm}$ & $30 \mathrm{~J} / \mathrm{cm}$ & $30 \mathrm{~J} / \mathrm{cm}$ & $\mathrm{N} / \mathrm{A}$ & $30 \mathrm{~J} / \mathrm{cm}$ & $30 \mathrm{~J} / \mathrm{cm}$ \\
\hline - Fimaporfin dose & $0.06 \mathrm{mg} / \mathrm{kg}$ & $0.25 \mathrm{mg} / \mathrm{k}$ & $0.25 \mathrm{mg} / \mathrm{kg}$ & & $0.25 \mathrm{mg} / \mathrm{kg}$ & $0.25 \mathrm{mg} / \mathrm{kg}$ \\
\hline RECIST outcomes at $3 / 6$ months & $\mathrm{SD} / \mathrm{SD}$ & & $\mathrm{SD} / \mathrm{PR}$ & & $\mathrm{PR} / \mathrm{SD}$ & \\
\hline $\begin{array}{l}\text { Survival, months from study } \\
\text { inclusion }\end{array}$ & 47 & & 50 (alive; April & & 14 & \\
\hline No. stent exchanges & & & & & & \\
\hline - Planned & 10 & & 17 & & 4 & \\
\hline - Unplanned & 4 & & 1 & & 4 & \\
\hline $\begin{array}{l}\text { Serious adverse events on study } \\
\text { (no., severity) }\end{array}$ & Cholangitis: 1 & e 3) & Cholangitis: 1 & ade 3) & $\begin{array}{l}\text { Cholangitis: } \\
1 \text { grade 2) }\end{array}$ & grade 3 \\
\hline - SAE related to $\mathrm{PCI}$ & No & & No & & 1 of 3 (see $C$ & \\
\hline $\begin{array}{l}\text { CA, cancer antigen; CEA, carcinoemb } \\
\text { in solid tumors; SD, stable disease; } \mathrm{P} \\
{ }^{1} \text { CA19-9 }(\mathrm{U} / \mathrm{mL} \text {, highest normal refe } \\
{ }^{2} \text { A second compassionate-use PCI pr }\end{array}$ & $\begin{array}{l}\text { nic antigen; NR } \\
\text { artial response; } \\
\text { ce among cent } \\
\text { edure was cond }\end{array}$ & $\begin{array}{l}\text { corded; } \mathrm{N} / \mathrm{A} \text {, not ap } \\
\text { erious adverse event } \\
\text { ), CEA (ng/mL, high } \\
1 \text { months after the }\end{array}$ & $\begin{array}{l}\text { cable; } \mathrm{PCI} \text {, photoc } \\
\text { reference }<3.8) \text {. } \\
\text { conducted in the }\end{array}$ & nical internalizati & ; RECIST, Respo & evaluation criteria \\
\hline
\end{tabular}



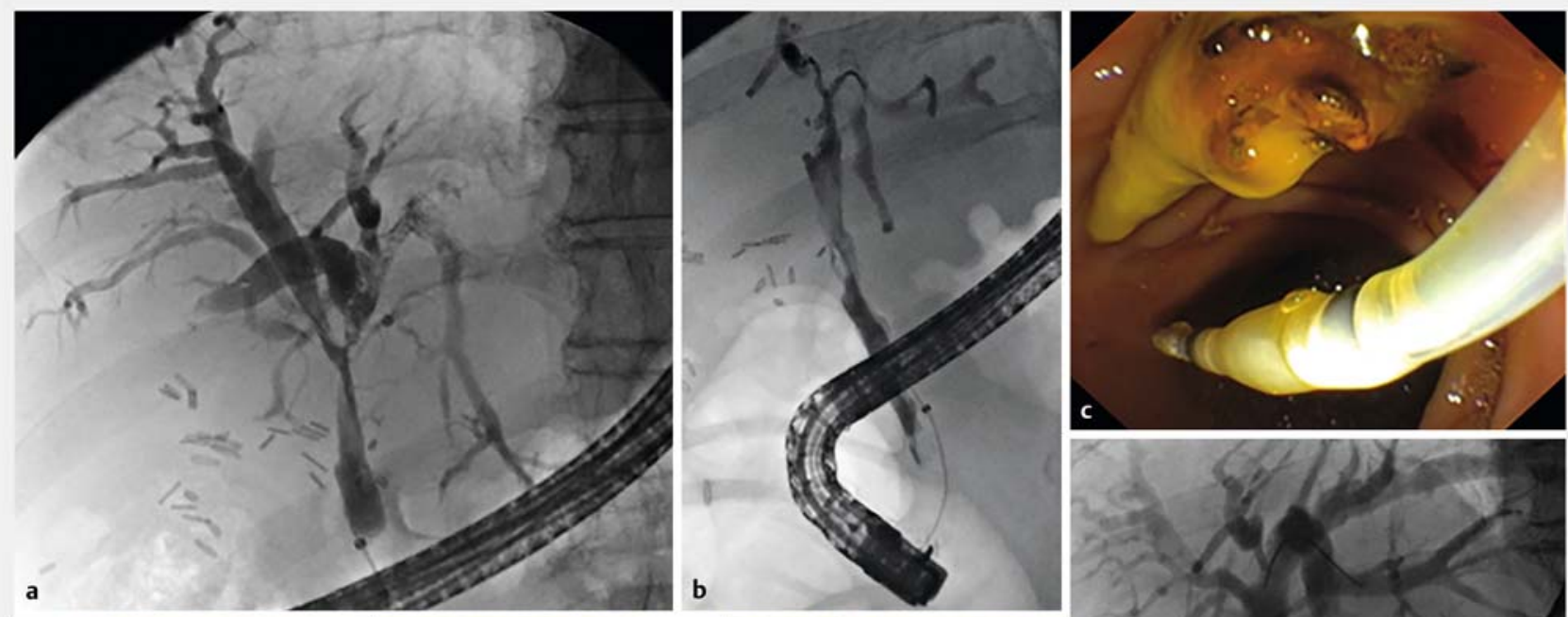

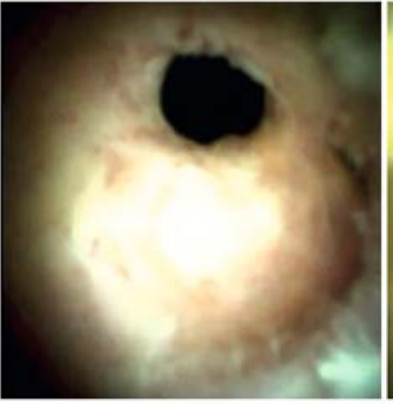

d Right hepatic duct

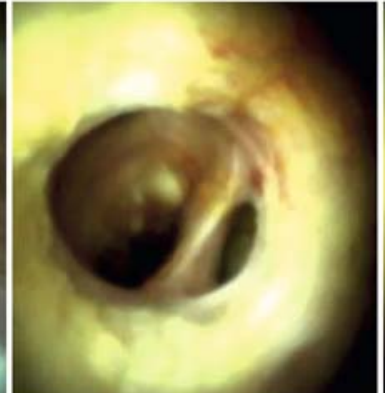

Left hepatic duct

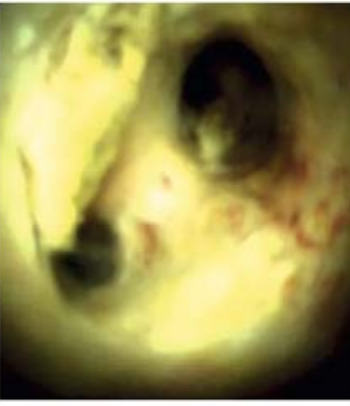

Bifurcation

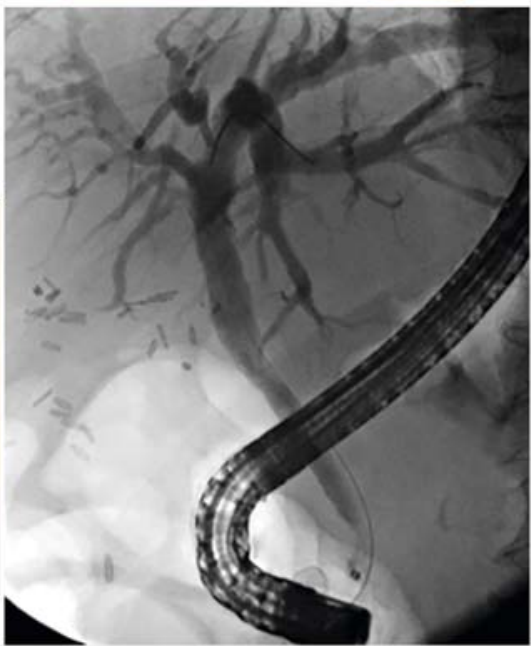

Fig. 2 Fluoroscopic cholangiograms from the patient in Case 3. a Before treatment, a 30-mm stenosis of the subhilar common bile duct (CBD) is seen, which extends $8 \mathrm{~mm}$ apically into both hepatic ducts. This finding corresponded to a $22.5 \mathrm{~mm} \times 20.2 \mathrm{~mm}$ tumor mass around the common bile duct observed on magnetic resonance imaging. $\mathbf{b}$ Four weeks after the second photochemical internalization procedure, the patient was admitted to the hospital with signs of cholangitis. After removal of the stents, an irregular stenosis of the CBD continuing into both hepatic ducts was seen, resembling the occlusion caused by the primary tumor. The stenosis consisted of a $4 \times 1 \mathrm{~cm}$ solid biliary cast of necrosis, which was visualized fluoroscopically and removed by balloon extraction. $\mathbf{c}$ The endoscopic appearance of the stenosis, consisting of the solid biliary cast of necrosis is seen. The bile duct was left without endoprostheses, as no occlusion remained. $\mathbf{d}$ Repeat cholangioscopy 1 week after removal of the necrotic mass. No stenosis and no dilatation of the intrahepatic bile ducts was detected fluoroscopically. The right and left hepatic ducts and the bifurcation are seen. Concomitantly, a partial response of the target lesion was seen at on prescheduled magnetic resonance imaging.

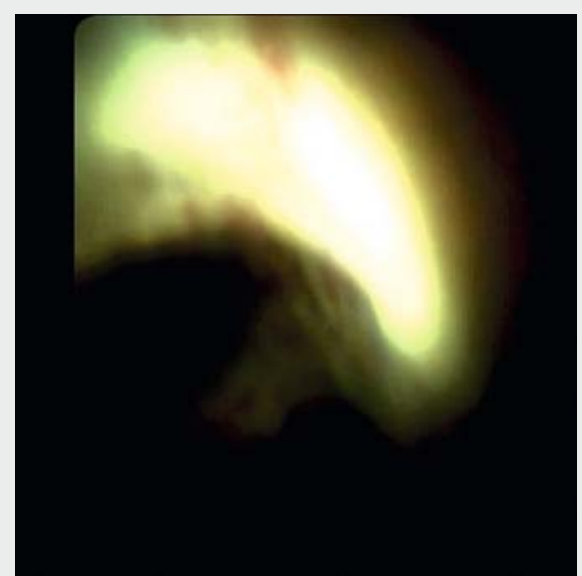

$\checkmark$ Video 1 Case 3. A video of the endoscopic findings. traction. MRI at 6 months demonstrated SD of the target lesion $(15.7 \mathrm{~mm} \times 14.7 \mathrm{~mm})$ around the CBD, and while this local treatment effect persisted, an unscheduled CT scan later revealed ascites and disseminated peritoneal spread. After two $\mathrm{PCl}$ procedures and three chemotherapy cycles, the patient died in April 2019, 17 months after the diagnosis of an unresectable perihilar cholangiocarcinoma.

\section{Discussion}

Unresectable perihilar CCA has a dismal prognosis, with an average survival of less than 12 months after diagnosis when treated with standard-of-care gemcitabine/cisplatin chemotherapy [3]. Locoregional treatments, most notably PDT, have been encouraging in halting disease progression when compared with biliary drainage alone. However, solid prospective data are limited on the combination of PDT with chemotherapy [4]. 
$\mathrm{PCl}$ provides a dual mechanism of action by combining the direct phototoxic, PDT-like effects with facilitation of the intracellular target reach of several cytotoxic agents [5]. In the patients presented herein with unresectable, perihilar CCA treated with $\mathrm{PCl}$ with gemcitabine combined with standard-of-care gemcitabine/cisplatin therapy, the clinical findings indicate that local tumor control was achieved. All of them had SD or partial remissions during the 6 months of the trial. The patients in Cases 1 and 2 both were treated with a single $\mathrm{PCl}$ procedure followed by eight standard chemotherapy cycles and they had progression-free survival of 17 and 27 months, respectively. In the patient in Case 1, a second "ad hoc" PCI treatment on biliary tumor progression at 20 months from treatment initiation seemed to induce an additional period of local tumor stabilization with a survival of 49 months from diagnosis. In Case 3, the patient's intraductal biliary stenosis was visually completely ablated by the treatment, and re-stenosis at follow-up was modest.

In Case 2, peritoneal spread was present. PCI and gemcitabine/cisplatin resulted in disease stabilization of the primary tumor, but also of metastatic sites in the peritoneal cavity. Systemic, distal (a.k.a. abscopal effect) responses to localized treatment have been reported with other combination regimens involving cryotherapy, radiation therapy, and immunotherapy, and also described after PDT [4, 7]. A similar immunological response mechanism induced by $\mathrm{PCl}$ treatment may possibly have influenced survival in this patient.

Two independent, recently published retrospective analyses found survival benefits by adding PDT to systemic chemotherapy in eCCA compared with chemotherapy alone [8,9]. Wu et al [8] not only found PDT to significantly improve 5-year overall survival compared with systemic treatment alone, but also that PDT as the only treatment provided had survival similar to chemotherapy treatment. In the dataset by Gonzalez-Carmona et al [9], PDT alone resulted in a higher median survival than chemotherapy alone (15 months vs 10 months). PDT was a significant, independent predictor of prolonged survival on multivariate analysis, interestingly most prominently in metastatic disease treated with PDT combined with chemotherapy compared with chemotherapy alone, and in further support of systemic effects from local therapies added to systemic.

This case series is limited to three subjects in a cohort of 23 patients included in the phase I/II PCI trial. Accordingly, it has no bearing on the overall safety and efficacy profile of $\mathrm{PCI}$ in eCCA and is not it intended to construe the compiled trial data and conclusions, yet to be published. However, the clinical findings in these subjects may indicate that local tumor control was induced by a single gemcitabine infusion combined with $\mathrm{PCl}$, reducing the pace of the otherwise difficult-to-treat hilar tumor progression and following liver failure. These observations are aligned with data in the literature suggesting that adding targeted, extrahepatic disease treatment may provide for better standard-of-care treatment results if more widely used, or rather, systematically incorporated as an adjunct to systemic therapies. Irrespective of systemic treatment, progression of the perihilar tumor growth is a hallmark and key factor for mor- tality. Furthermore, the observations, such as in Case 1, indicate the potential applicability of $\mathrm{PCl}$ in palliative treatment.

\section{Conclusion}

Treatment of locally advanced perihilar CCA with PCI followed by gemcitabine/cisplatin represents a treatment modality with a potential to prolong local tumor control, which needs to be verified. If so, $\mathrm{PCl}$ with gemcitabine may support the adherence to and completion of systemic treatment regimens and possibly induce immune response sporadically. A global randomized trial to investigate the role of $\mathrm{PCl}$ combined with gemcitabine/ cisplatin in the treatment of unresectable perihilar and distal CCA has now been initiated.

\section{Acknowledgements}

This work was supported by PCI Biotech AS (Oslo, Norway) through the sponsorship of study PCI A202/12 (EudraCT No 2012-002888-10; NCT01900158).

\section{Competing interests}

Dr. Olivecrona is a consultant for PCI Biotech AS. Drs. Dechêne, Kasper, Schirra, and Trojan participated as investigators in the study $\mathrm{PCI}$ A202/12.

\section{References}

[1] Banales JM, Cardinale V, Carpino G et al. Expert consensus document: Cholangiocarcinoma: current knowledge and future perspectives consensus statement from the European Network for the Study of Cholangiocarcinoma (ENS-CCA). Nat Rev Gastroenterol Hepatol 2016; 13: 261-280

[2] Esnaola NF, Meyer JE, Karachristos A et al. Evaluation and management of intrahepatic and extrahepatic cholangiocarcinoma. Cancer 2016; 122: 1349-1369

[3] Valle JW, Furuse J, Jitlal M et al. Cisplatin and gemcitabine for advanced biliary tract cancer: a meta-analysis of two randomised trials. Ann Oncol 2014; 25: 391-398

[4] Agostinis P, Berg K, Cengel KA et al. Photodynamic therapy of cancer: An update. CA Cancer J Clin 2011; 61: 250-281

[5] Selbo PK, Weyergang A, Hogset A et al. Photochemical Internalisation provides time-and space-controlled endolysosomal escape of therapeutic molecules. J Controlled Release 2010; 148: 2-12

[6] Sultan AA, Jerjes W, Berg K et al. Disulfonated tetraphenyl chlorin (TPCS2a)-induced photochemical internalisation of bleomycin in patients with solid malignancies: a phase 1, dose-escalation, first-inman trial. Lancet Oncol 2016; 17: 1217-1229.

[7] Ngwa W, Irabor OC, Schoenfeld JD et al. Using immunotherapy to boost the abscopal effect. Nat Rev Cancer 2018; 18: 313-322

[8] Wu L, Merath K, Farooq A et al. Photodynamic therapy may provide a benefit over systemic chemotherapy among non-surgically managed patients with extrahepatic cholangiocarcinoma. J Surg Oncol 2019: $1-8$

[9] Gonzalez-Carmona MA, Bolch M, Jansen C et al. Combined photodynamic therapy with systemic chemotherapy for unresectable cholangiocarcinoma. Aliment Pharmacol Ther 2019; 49: 437-447 